Adipositas - Ursachen, Folgeerkrankungen, Therapie 2021; 15(03): 154

DOI: $\underline{10.1055 / s-0041-1735681}$

Abstracts

Wiesbaden: Adipositas-Kongress 2021

\title{
80 Effekte von Lebensstilinterventionen auf Körpergewicht und Fettmasse bei älteren Menschen mit Adipositas - eine systematische Übersichtsarbeit mit Netzwerkmetaanalysen
}

\section{Gabriel Torbahn}

1 Institut für Biomedizin des Alterns, Friedrich-Alexander-Universität Erlangen-Nürnberg, Nürnberg, Deutschland

\section{Daniel Schoene}

2 Institut für Medizinische Physik, Friedrich-Alexander-Universität Erlangen-Nürnberg, Erlangen, Deutschland

\section{Isabel Galicia Ernst}

1 Institut für Biomedizin des Alterns, Friedrich-Alexander-Universität Erlangen-Nürnberg, Nürnberg, Deutschland

Lukas Schwingshackl

3 Institut für Evidenz in der Medizin, Universitätsklinikum Freiburg, Medizinische Fakultät, AlbertLudwigs-Universität Freiburg, Freiburg, Deutschland

\section{Gerta Rücker}

4 Institut für Medizinische Biometrie und Statistik, Universitätsklinikum Freiburg, Medizinische Fakultät, Albert-Ludwigs-Universität Freiburg, Freiburg, Deutschland

\section{Helge Knüttel}

5 Universitätsbibliothek Regensburg, Universität Regensburg, Regensburg, Deutschland

\section{Wolfgang Kemmler}

2 Institut für Medizinische Physik, Friedrich-Alexander-Universität Erlangen-Nürnberg, Erlangen, Deutschland

\section{Cornel Sieber}

1 Institut für Biomedizin des Alterns, Friedrich-Alexander-Universität Erlangen-Nürnberg, Nürnberg, Deutschland

6 Departement Medizin, Kantonsspital Winterthur, Winterthur, Schweiz

\section{JohnA Batsis}

7 Division of Geriatric Medicine, School of Medicine, University of North Carolina at Chapel Hill, Chapel Hill, USA 
8 Department of Nutrition, Gillings School of Global Public Health, University of North Carolina at Chapel Hill, Chapel Hill, USA

\section{Dennis T Villareal}

9 Division of Endocrinology, Diabetes and Metabolism, Baylor College of Medicine, Houston, USA

\section{Nanette Ströbele-Benschop}

10 Institut für Ernährungsmedizin, Universität Hohenheim, Stuttgart-Hohenheim, Deutschland

\section{Dorothee Volkert}

1 Institut für Biomedizin des Alterns, Friedrich-Alexander-Universität Erlangen-Nürnberg, Nürnberg, Deutschland

\section{Eva Kiesswetter}

1 Institut für Biomedizin des Alterns, Friedrich-Alexander-Universität Erlangen-Nürnberg, Nürnberg, Deutschland

\section{Zusammenfassung}

Multimodale Lebensstilinterventionen, besonders $K R+A T+K T+V M$, führen bei älteren Personen mit Adipositas zu einer (signifikanten) Reduktion des KG und der FM.

\section{Einleitung}

Die Adipositasprävalenz in Deutschland steigt bei älteren ( $\geq 65$ Jahre) Personen und beträgt derzeit ca. 30\%. Auch im höheren Alter gelten Lebensstilinterventionen als erste Therapieoption. Ziel der Arbeit war die systematische Zusammenfassung der Effekte von Lebensstilinterventionen auf das Körpergewicht (KG) und die Fettmasse (FM) bei älteren Personen mit Adipositas.

\section{Material und Methodik}

Nach einer Literaturrecherche in 6 Datenbanken sichteten 2 Gutachter*innen unabhängig voneinander Titel/Abstracts und Volltexte, bewerteten das Verzerrungsrisiko (Cochrane Risk of Bias 2.0 Tool) und extrahierten Daten. Einschlusskriterien waren: i) randomisiert-kontrollierte Studien (RCTs), ii) zuhause lebende ältere Personen (Mindestalter $\geq 60 \& \varnothing$-Alter $\geq 65$ Jahre) iii) Adipositas (BMI $\geq 30 \mathrm{~kg} / \mathrm{m}^{2}$, Taillenumfang $\geq 88 / 102 \mathrm{~cm}$, Fettanteil $\geq 35 / 25 \%$ für Frauen/Männer; oder abweichende validierte Schwellenwerte (Asien)), iv) Lebensstilinterventionen (Kalorienrestriktion (KR), eiweißreiche Diät (ED), Ausdauer- (AT) oder Krafttraining (KT), Verhaltensmodifikation (VM)) und deren Kombinationen sowie v) Dauer von $\geq 12$ Wochen. Um Interventionseffekte auf das KG und die FM statistisch zusammenzufassen, wurden Netzwerkmetaanalysen (NMA) durchgeführt und MD $[95 \%-\mathrm{Cl}]$ gepoolt. 


\section{Ergebnisse}

Dreiundzwanzig RCTs ( $n=1941 ; \varnothing$-Alter 68,5 Jahre, 17 Knoten, 35 paarweise Vergleiche) wurden in die NMA zum KG eingeschlossen. Alle Interventionen reduzierten das KG, wobei sich die Interventionen $\mathrm{KR}+\mathrm{AT}+\mathrm{VM}(-10,29[-17,62 ;-2,95] \mathrm{kg})$, KR+AT+KT+VM $(-8,64[-14,70 ;-2,59] \mathrm{kg})$, $\mathrm{KR}+\mathrm{KT}+\mathrm{VM}(-8,55[-16,53 ;-0,57] \mathrm{kg}), \mathrm{KR}+\mathrm{ED}+\mathrm{VM}(-6,39[-7,65 ;-5,14] \mathrm{kg})$ und VM $(-1,30[-2,44 ;-$ $0,16] \mathrm{kg}$ ) signifikant von der Kontrolle unterschieden, während alle anderen Kombinationen keine signifikanten Effekte erzielten. Die NMA zur FM schloss 20 RCTs ein ( $n=1349 ; \varnothing$-Alter 68,8 Jahre; 17 Knoten, 37 paarweise Vergleiche). Im Vergleich zur Kontrolle führten alle Interventionen, die eine KRKomponente enthielten, zu einer Reduktion der FM, wobei die Kombination aus KR+AT+KT+VM den einzigen signifikanten Effekt zeigte $(-6,96[-11,93 ;-1,99] \mathrm{kg})$. Alle anderen Interventionen wiesen geringere und nicht-signifikante Effekte auf, bis auf VM allein, die eine signifikante Reduktion von $-1,10[-2,08 ;-0,12] \mathrm{kg}$ zeigte. Für beide Netzwerke unterschieden sich direkte und indirekte Evidenz nicht signifikant. Das Verzerrungsrisiko war für 22/28 RCTs bedenklich bis hoch. 